\title{
Generation of 20-Gb/s RZ-DQPSK Signal using a Directly Modulated Chirp Managed Laser
}

\author{
Wei Jia, Jing Xu, Zhixin Liu, Chun-Kit Chan and Lian-Kuan Chen \\ Department of Information Engineering, The Chinese University of Hong Kong, Shatin, N.T., Hong Kong SAR \\ jw009@ie.cuhk.edu.hk
}

\begin{abstract}
We generate 20-Gb/s return-to-zero differential quadrature phase-shift-keying (RZDQPSK) signal using a directly modulated chirp managed laser (CML), without requiring any differential encoder and optical phase modulator.

(C)2011 Optical Society of America

OCIS codes: (060.2330) Fiber optics communications; (060.2360) Fiber optics links and subsystems
\end{abstract}

\section{Introduction}

Return-to-zero differential quadrature phase-shift-keying (RZ-DQPSK) modulation format has attracted much attention due to the advantages of increased spectral efficiency and high robustness towards fiber nonlinearities [1]. In [2], 2.67-Gb/s return-to-zero differential phase-shift-keying (RZ-DPSK) signal with $2^{7}-1$ pseudo-random bit sequence (PRBS) data was obtained using a directly modulated chirp managed laser (CML), which comprises a distributed feedback (DFB) laser and a passive optical filter [3]. The CML was driven by a three-level RZ signal.

In this paper, we demonstrate a novel scheme to generate 20-Gb/s RZ-DQPSK signal using a commercially available 10-Gb/s CML. The two input data streams are firstly encoded in binary Inverse-Return-to-Zero (IRZ) format with different driving voltages and then combined by a passive RF combiner to generate a four-level IRZ signal to drive the CML. The transmitter cost can be significantly reduced as no differential encoder and optical phase modulator (PM) are required. This scheme could be generalized to generate the $M$-ary RZ-DPSK signal, where all the $\log _{2} M$ data streams are in IRZ format with different driving voltages and combined to drive the CML.

\section{Operation Principle}

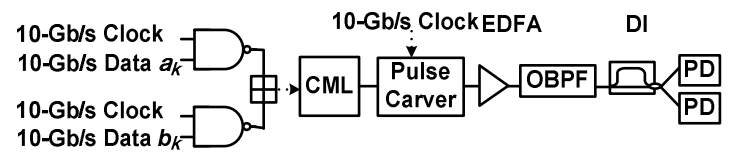

(a)
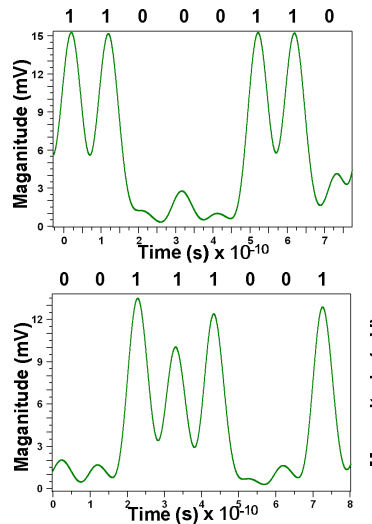

(b)

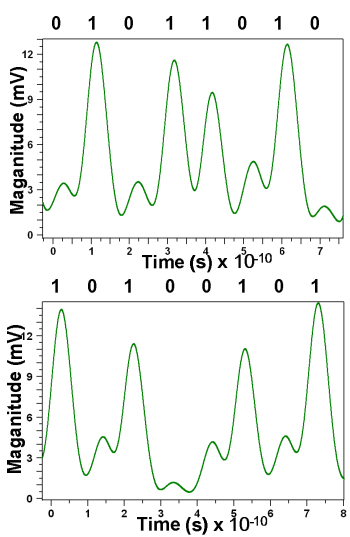

(c)

Fig. 1 (a) Proposed scheme of RZ-DQPSK generation system, (b)

Demodulated output data $p_{k}$ and (c) data $q_{k}$ at two ports of the DI

Fig. 1 (a) depicts the proposed scheme of 20-Gb/s RZ-DQPSK signal generation system using a CML. The transmitter consists of two IRZ encoders, a passive RF combiner, a CML and a pulse carver. Fig.2 illustrates the operation principle through the intensity, chirp and phase characteristics of output signals of the IRZ encoders, RF combiner, DFB laser (inside CML) and pulse carver. Two IRZ-shaped data sequences $a_{k}=00111001$ and $b_{k}=10011100$ with a duty cycle of $50 \%$ are generated, via two commercial logic NAND gates separately, before 


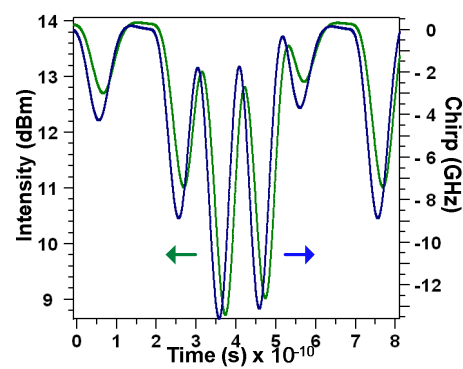

(a)

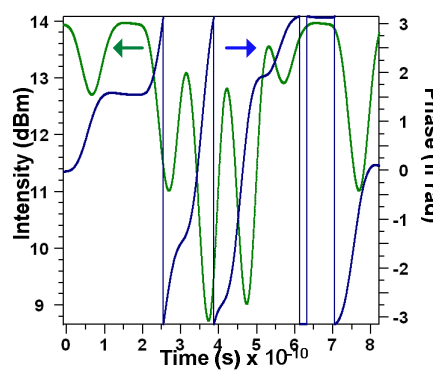

(b)

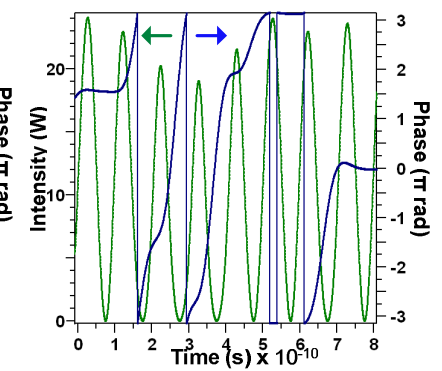

(c)

Fig. 3 Simulated (a) chirp profile of DFB laser output signal, (b) phase profile of DFB laser output signal and (c) pulse carver output signal

being combined to directly modulate the CML. The driving voltages of the two data sequences are $V_{p p}$ and $0.5 V_{p p}$ respectively. The laser is biased high above the threshold with the benefits of high output power, wide modulation bandwidth and suppression of transient chirp [3]. The driving voltage $V_{p p}$ is adjusted to induce adiabatic chirp of $\Delta f=1 / T$, which generates phase shift $\Delta \phi=2 \pi \int_{0}^{\mathrm{T} / 2} \Delta f(t) d t=2 \pi \times 1 / T \times T / 2=\pi$ during the first half-bit period of " 10 " symbol. $T$ denotes the symbol period. Thus the phase shifts for the two-bit input $a_{k} b_{k}$ of " 00 ", " 01 ", " 10 ", and " 11 "

\begin{tabular}{|ccc|ccc|}
\hline $\begin{array}{c}\text { Input Data Phase Diff. } \\
a_{k} b_{k}\end{array}$ & $\Delta \phi$ & $\begin{array}{c}\text { Demodulated Output data } \\
p_{k} q_{k}\end{array}$ \\
\hline 00 & 0 & 11 \\
01 & $0.5 \pi$ & 01 \\
10 & $\pi$ & 00 \\
11 & $1.5 \pi$ & 10 & $\begin{array}{cccc}\text { Input data } \\
a_{k} b_{k}\end{array}$ & $\begin{array}{c}\text { Phase Diff. } \\
0\end{array}$ & $\begin{array}{c}\text { Demodulated Output Data } \\
p_{k} q_{k}\end{array}$ \\
01 & $0.5 \pi$ & 00 \\
010 & $1.5 \pi$ & 01 \\
11 & 0 & 10 \\
11 & 0 & 11 \\
\hline
\end{tabular}

(a)

(b)

\begin{tabular}{|ccccc|}
\hline $\begin{array}{c}\text { Input Data } \\
a_{k} b_{k}\end{array}$ & Precoding Operation & $\begin{array}{c}\text { Precoded Data } \\
\boldsymbol{a}_{k}{ }^{*} b_{k}{ }^{*}\end{array}$ & $\begin{array}{c}\text { Phase Diff. } \\
\Delta \phi\end{array}$ & $\begin{array}{c}\text { Demodulated Output data } \\
\boldsymbol{p}_{k} \boldsymbol{q}_{k}\end{array}$ \\
\hline 00 & $\boldsymbol{a}_{k}{ }^{*}=\bar{b}_{k}$ & 10 & $\pi$ & 00 \\
01 & 01 & $0.5 \pi$ & 01 \\
10 & $\boldsymbol{b}_{k}{ }^{*}=a_{k} \oplus \boldsymbol{b}_{k}$ & 11 & $1.5 \pi$ & 10 \\
11 & 00 & 0 & 11 \\
\hline
\end{tabular}

(c)

Fig. 4 The relationships among input data, phase difference and demodulated output data of (a) CML based RZ-DQPSK signal, (b) PM based RZ-DQPSK signal and (c) precoding at the transmitter site instead of decoding at the receiver site for CML based RZ-DQPSK signal.

are $0,0.5 \pi, \pi$, and $1.5 \pi$, respectively. The maximum chirp value required for CML to generate 20-Gb/s RZDQPSK signal is $15 \mathrm{GHz}$. The relative spectral locations of the laser and filter are detuned to change the signal extinction ratio (ER). The pulse carver with a duty cycle of 50\% carves the second half-bit of the phase-modulated signal, thus generating a RZ-DQPSK signal. Fig. 3 shows the simulated intensity, chirp and phase profiles of the DFB laser and pulse carver output signals. The phase modulation is intrinsically differentially encoded. Differential encoder and PM are not needed. At the receiver, the phase differences $\Delta \phi=0,0.5 \pi$, and $1.5 \pi$ corresponding to the two-bit input $a_{k} b_{k}$ of " 00 ", " 01 ", " 10 ", and " 11 " are demodulated into two-bit output $p_{k} q_{k}$ of " 11 ", " 01 ", " 00 ", and "10", respectively, shown via simulation results in Fig. 1 (b) and Fig. 1 (c). Fig. 4 (a) and (b) show that the relationships among input data, phase difference, and demodulated output data of CML and PM based RZ-DQPSK signals are different [5]. Therefore, decoding of $a_{k}=\overline{q_{k}}$ and $b_{k}=p_{k} \oplus q_{k}$ is necessary to retrieve data $a_{k}$ and data $b_{k}$ correctly at the receiver for CML based RZ-DQPSK signal. Fig. 4 (c) shows that the complementary and exclusiveor operations can also be implemented at the transmitter site instead of the receiver site.

\section{Experiment and Results}

We have experimentally demonstrated the proposed 20-Gb/s RZ-DQPSK generation system using a CML, as shown in Fig. 1 (a). We used a standard CML module (Finisar DM80-01) in the experiment, which is the same as the one used in [2]. The input impedance, threshold current and FM efficiency of the DFB laser are $50 \mathrm{ohms}, 25 \mathrm{~mA}$ and $\sim 0.24 \mathrm{GHz} / \mathrm{mA}$, respectively. The filter in DM80-01 has a $3 \mathrm{~dB}$ bandwidth of $\sim 11 \mathrm{GHz}$ and an average slope of $\sim 1.5$ $\mathrm{dB} / \mathrm{GHz}$. Two 10-Gb/s IRZ data streams with respective driving voltages of $\sim 1.6 \mathrm{~V}$ and $\sim 0.8 \mathrm{~V}$ were combined by a passive RF combiner before directly modulating the DFB laser using $2^{7}-1$ PRBS. The laser was biased at $85 \mathrm{~mA}$. The central wavelength of signal after the filter was $1555.47 \mathrm{~nm}$. A Mach-Zehnder intensity modulator (MZM) driven by a $10-\mathrm{GHz}$ clock was used as the pulse carver. The output power after the pulse carver was $\sim-0.5 \mathrm{dBm}$. An erbium-doped fiber amplifier (EDFA) was inserted at the receiver to boost optical signal power. A tunable optical band pass filter (OBPF) with $\sim 1 \mathrm{~nm}$ bandwidth was set after the EDFA to eliminate amplified spontaneous emission (ASE) noise. The generated RZ-DQPSK signal was demodulated by a 1-bit optical delay interferometer (DI) before being detected by a photo-detector. The phase difference between the two arms of DI was adjusted by controlling temperature to demodulate data $a_{k}$ and data $b_{k}$, respectively. 


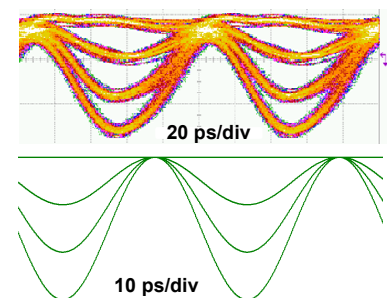

(a)

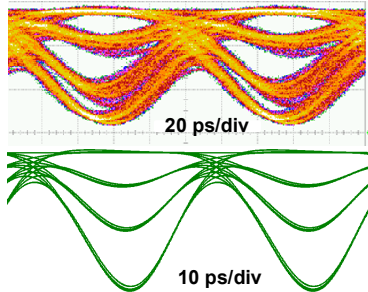

(b)

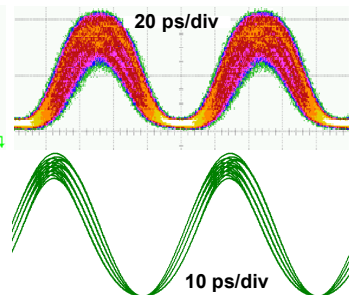

(c)

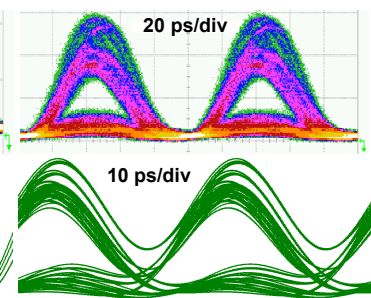

(d)

Fig. 5 Experimental (upper) and corresponding simulated (lower) eye diagrams of (a) signal after the RF combiner, (b) signal after the CML, (c) RZ-DQPSK signal after the pulse carver and (d) demodulated data $a_{k}$ signal at one port of the DI
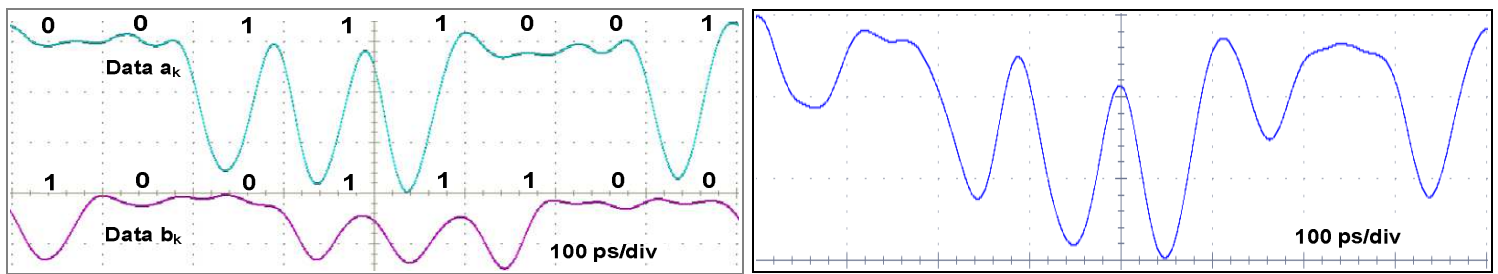

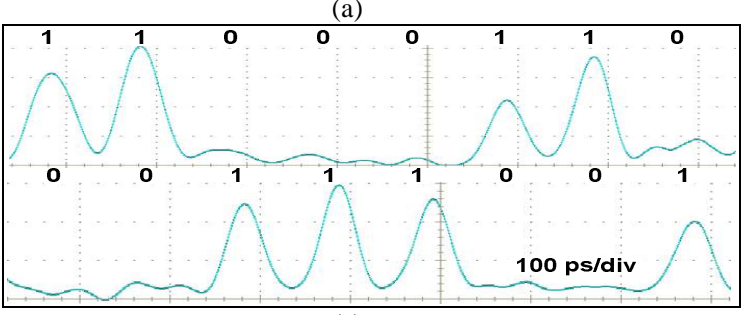

(c)

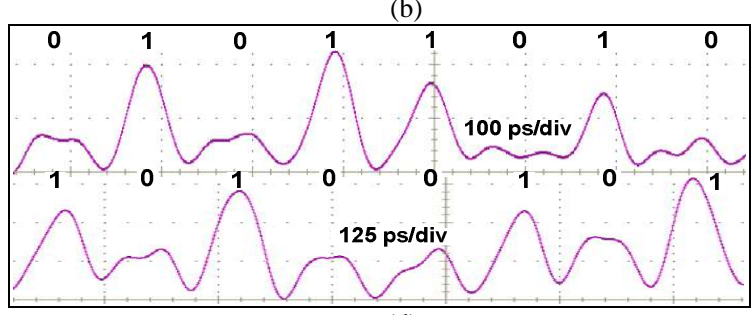

(d)

Fig. 6 Waveform traces for signals (a) of data $a_{k}$ and $b_{k}$, (b) after the RF combiner, (c) of demodulated data $p_{k}$ and (d) of demodulated data $q_{k}$.

Fig. 5 shows the experimental (upper) and simulated (lower) back-to-back (BtB) eye diagrams of the signals after the RF combiner, CML, pulse carver and demodulated data $a_{k}$ signal at one port of the DI. The multiple lines of RZ-DQPSK signal shown in Fig. 5 (c) are due to the imperfect driving signal and limited modulation response of the DFB laser [4] in CML as shown in Fig. 5 (a) and (b), respectively. Fig. 6 shows the waveform traces measured by a real-time oscilloscope. Two data sequences $a_{k}=00111001$ and $b_{k}=10011100$ were applied to the transmitter. The subtraction of the bottom and top demodulated waveforms, shown in Fig. 6 (c), would correctly regenerate the input bit sequence $a_{k}$. Decoding of $b_{k}=p_{k} \oplus q_{k}$ would also regenerate the input bit sequence $b_{k}$, as shown in Fig. 6 (c) and (d). It is illustrated that no differential encoding of the original data is required. The uneven marks and fluctuating spaces of demodulated signals shown in Figs. 6(c) and (d) are attributed to the imperfect driving signal shown in Fig. 5 (a) and Fig. 6 (b), limited modulation response and chirp value of the DFB laser. Since decoding of $b_{k}=p_{k} \oplus q_{k}$ was not performed here, only the BtB bit-error-rate (BER) performance of demodulated RZ-DQPSK data $a_{k}$ signal at one port of the DI was measured. The BtB receiver sensitivity at BER $=10^{-9}$ for CML based RZ-DQPSK signal was $-15.31 \mathrm{dBm}$. The corresponding eye diagrams are shown in Fig. 5 (d).

\section{Conclusions}

We propose and experimentally demonstrate a simple and cost-effective 20-Gb/s RZ-DQPSK transmitter using a directly modulated CML, without requiring any differential encoder and PM. This scheme could be generalized to generate $M$-ary RZ-DPSK signal.

\section{References}

[1] Christoph Wree et. al., "RZ-DQPSK format with high spectral efficiency and high robustness towards fiber nonlinearities," ECOC 2002, Paper 9.6.6.

[2] James Franklin et. al., "Generation of RZ-DPSK using a chirp-managed laser (CML),” OFC/NFOEC 2008, JWA67.

[3] Yasuhiro Matsui et. al., "Chirp-Managed Directly Modulated Laser (CML)," PTL, 18, 385-387 (2006).

[4] R. S. Vodhanel, et. al., "Ten-to-twenty gigabit-per-second modulation performance of $1.5-\mu \mathrm{m}$ distributed feedback lasers for frequency-shiftkeying systems," JLT, 7, 1454-1460 (1989).

[5] Keang-Po Ho, "Phase-Modulated Optical Communication Systems," Springer, New York (2005). 